\title{
An Open Source Software Directory for Aeronautics and Space
}

Andreas Schreiber, Michael Meinel, Tobias Schlauch German Aerospace Center (DLR)

Roberto Galoppini

SourceForge

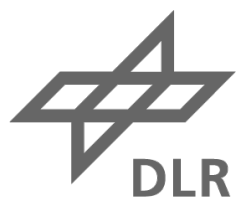




\section{Outline}

- DLR

- Software at DLR

- Software Catalogue

- Allura

- DLR Software Portal

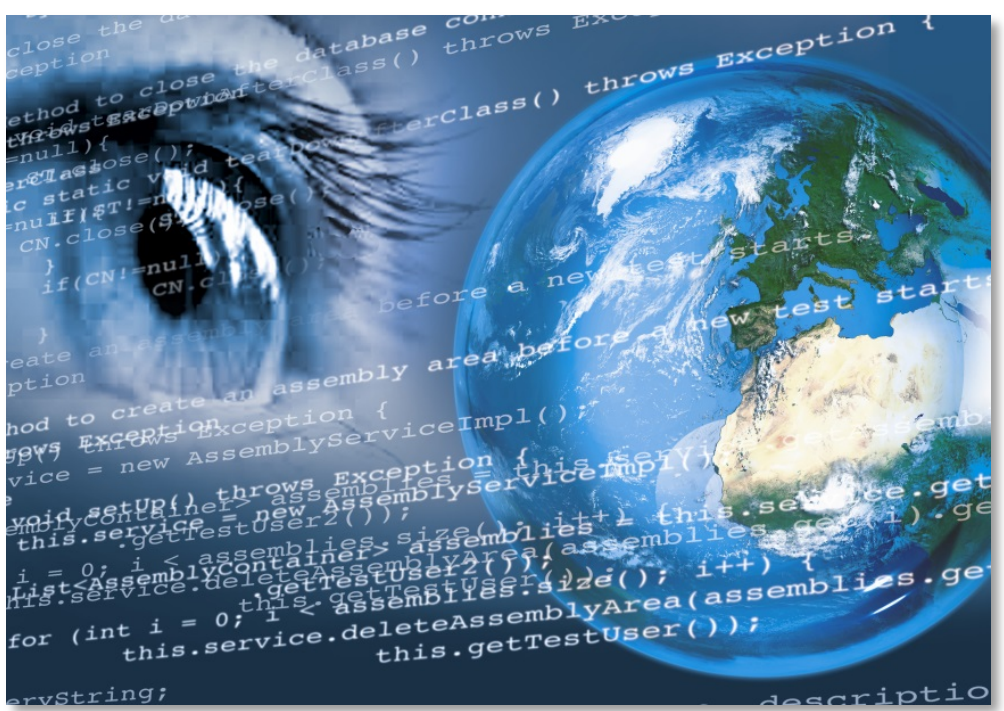




\section{DLR}

\section{German Aerospace Center}

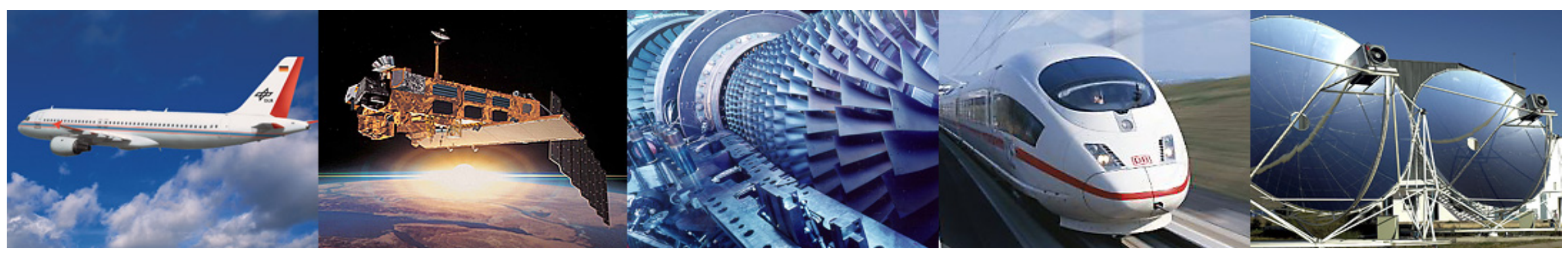

- Research Institution

- Space Agency

- Project Management Agency

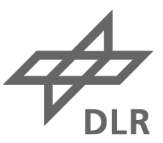




\section{DLR \\ Locations and employees}

8000 employees across

33 institutes and facilities at

-16 sites.

Offices in Brussels, Paris, Tokyo and Washington.

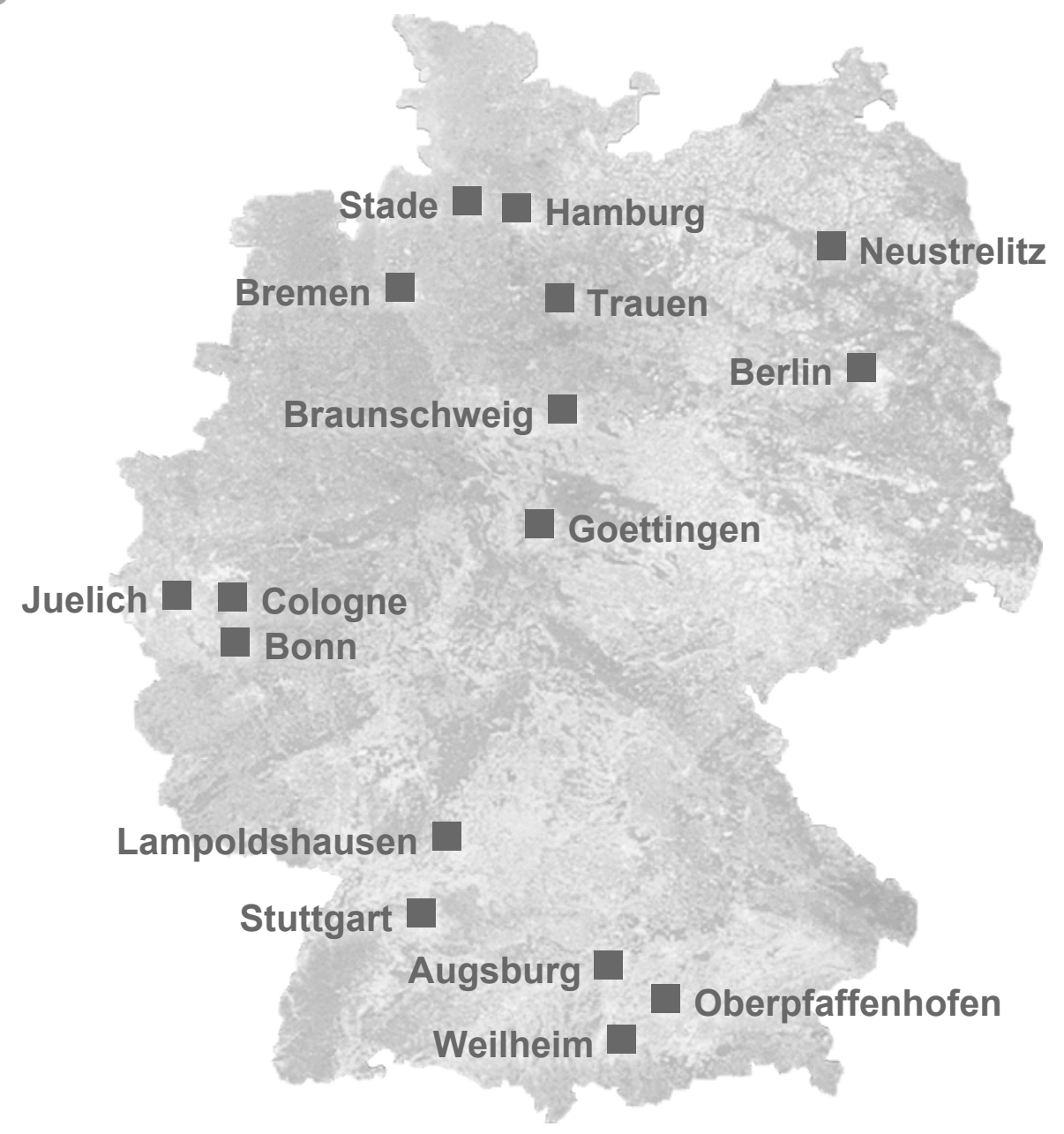




\section{DLR}

Research Areas

Major research areas of DLR institutes

- Aeronautics

- Space

- Transportation

- Energy

- Security

Software research and development

- Simulation and Software Technology division
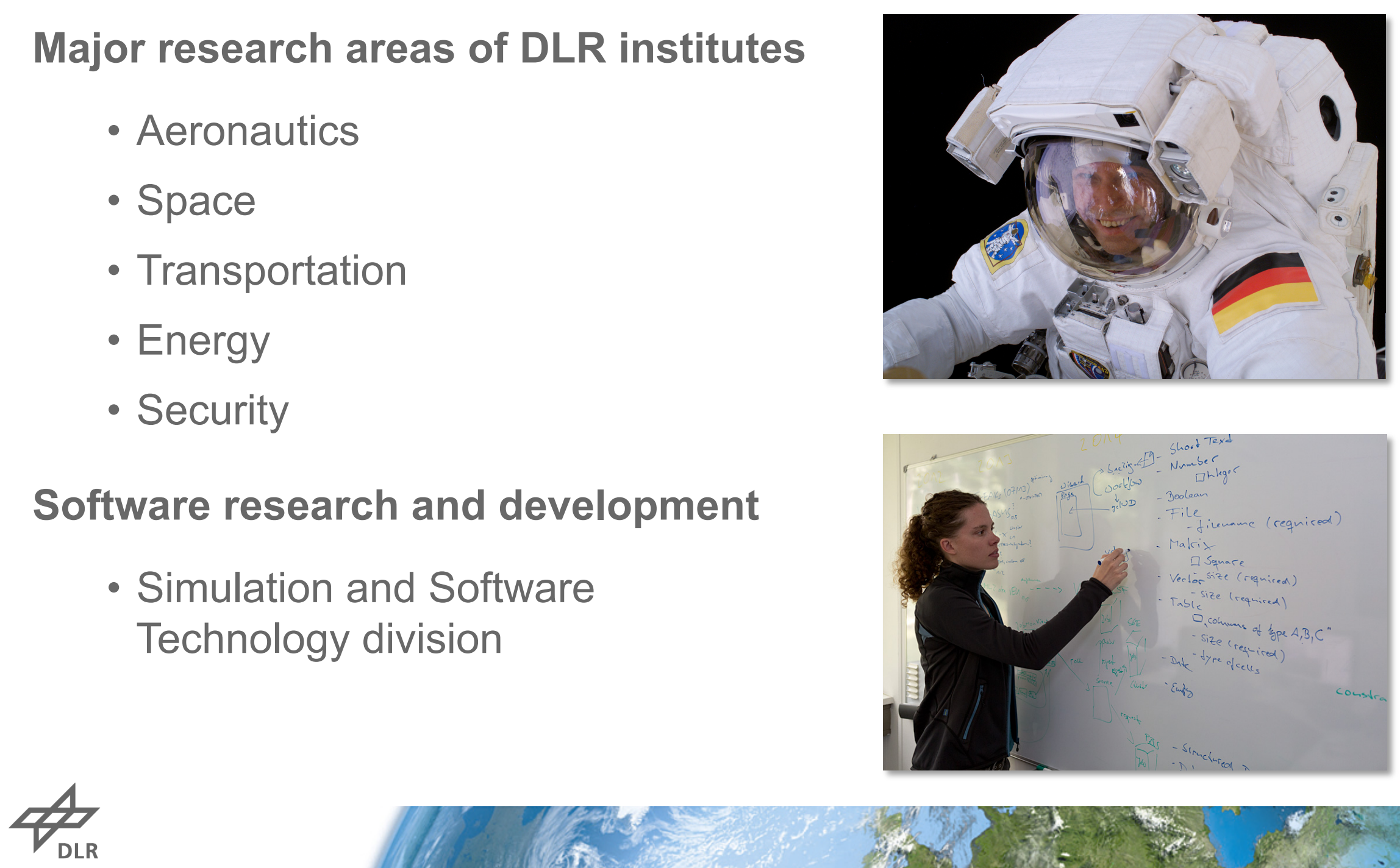


\section{Software at DLR Size and Amount}

Some numbers...

- More than 1200 employees are developing software

- More than 100 Million EURO personnel costs per year

- DLR is one of Germany largest software developers
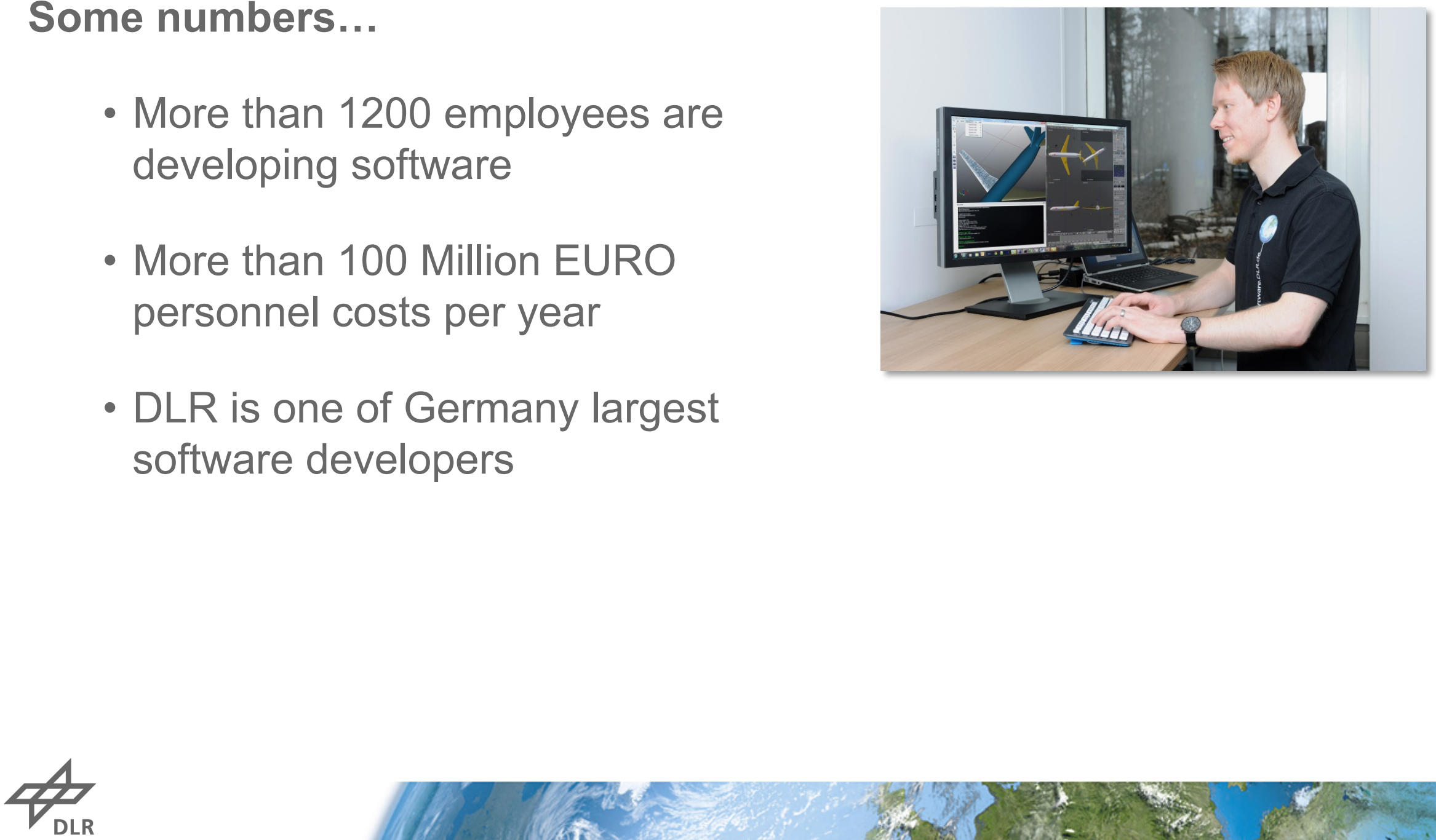


\section{Software at DLR}

\section{Typical Software in Space and Aerospace}

\section{Simulation Software}

- High performance computing and scientific computing

Mission-critical software

- Real-time | embedded | decision making software

\section{Supporting software}

- Data | Workflow | Knowledge management

Administrative software

- Web-based intranet software for accounting and project management

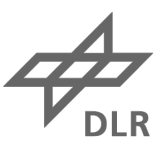




\section{Software at DLR Characteristics}

- Most software developed at DLR is non-standard software

- Often very special and specific requirements

- A great many number of software projects

- Open Source or proprietary software licenses

- Overview of existing software is extremely difficult

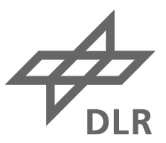




\section{Example}

\section{Future Aircraft Design}

Complex task with many involved scientific and engineering disciplines
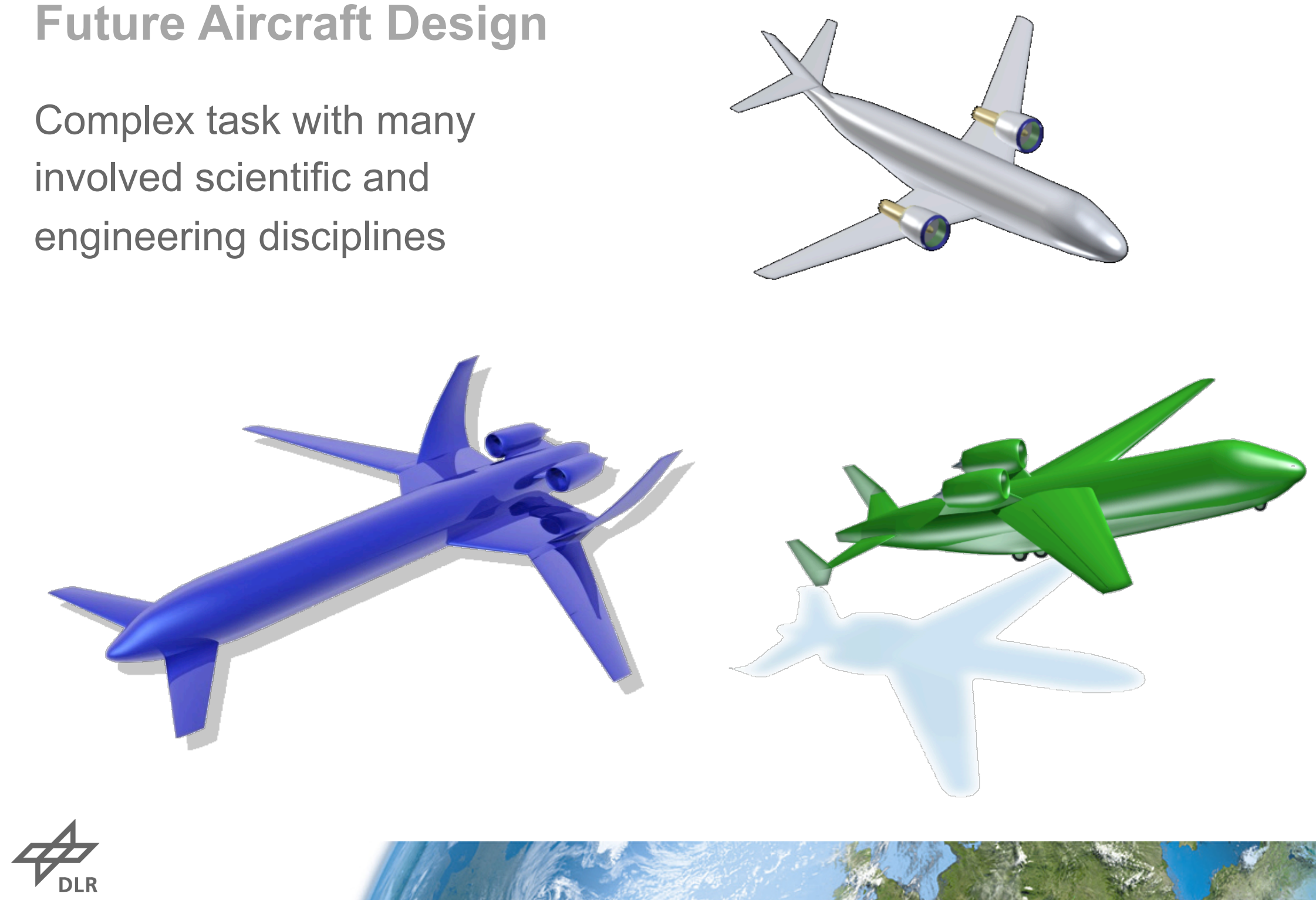


\section{Future Aircraft Design}

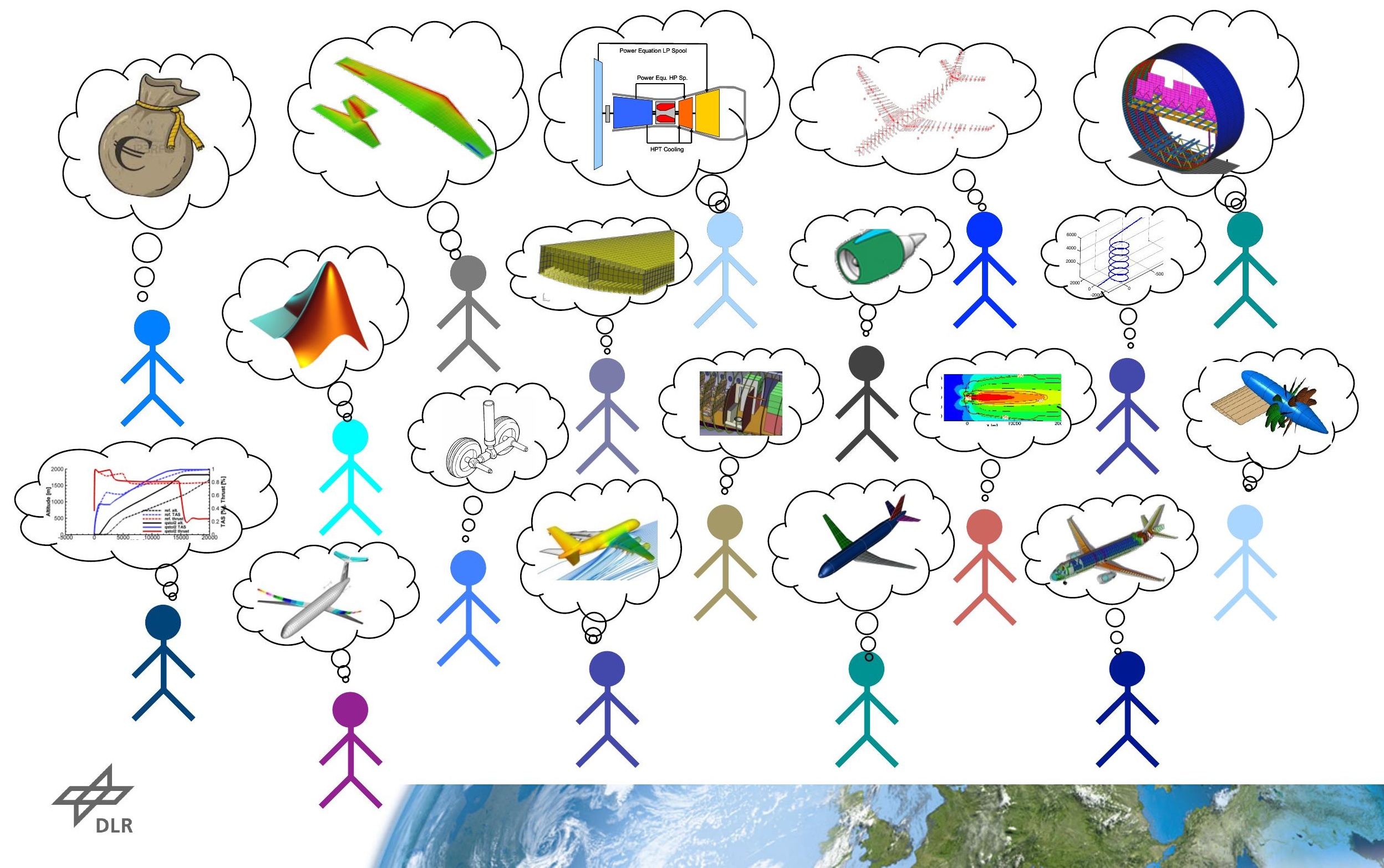




\title{
Software Engineering Strategy Dealing with DLRs Software Characteristics
}

\author{
Methods and Tools
}

- Development processes tailored for scientists, documentation via Web-based tools

- Development tools seamlessly integrated with working environment

- Tools are available and accessible easily via intranet for every employee

- Standard trainings offered for most important tool chains and software technologies

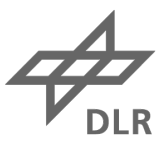




\section{Software Engineering Strategy Knowledge Management}

Exchange of knowledge and information

- Network of software engineering representatives

- Information sharing via intranet and workshops

- Wiki for documentation and collaboration

- Question \& Answer system (such as Stack Overflow)

- Software catalogue

Disclaimer: This list is intentionally not complete!

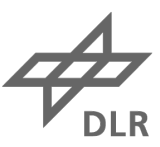




\section{Software Catalogue Goal and Essential Requirements}

Intention and goal

- Employees can get an overview of all software software packages, tools, and products developed at DLR

- To prevent double development of software

\section{Essential requirements}

- Searching for existing software

- Browsable directory of all software

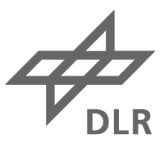




\section{Software Catalogue Major Requilrements}

\section{Technical requirements}

- Web-based

- Access control

- Basic project information

- Tagging

- Screenshots and diagrams

- Public page

- Code hosting

- Collaboration and documentation

- Commenting and rating

- Social media integration

- Scalability

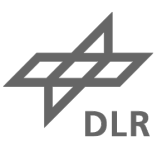




\section{Software Catalogue}

\section{First Version}

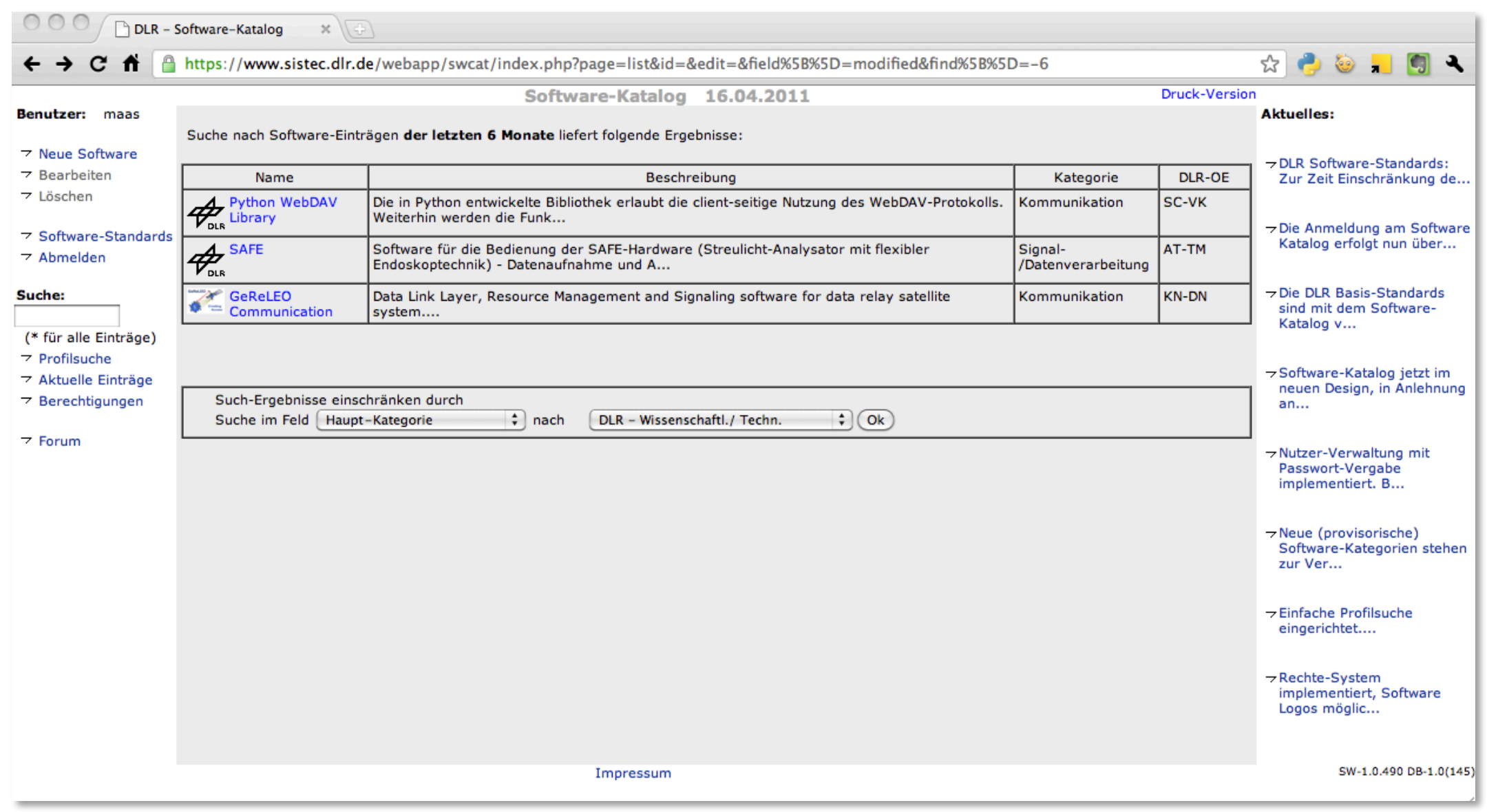




\section{Software Catalogue}

\section{First Version}

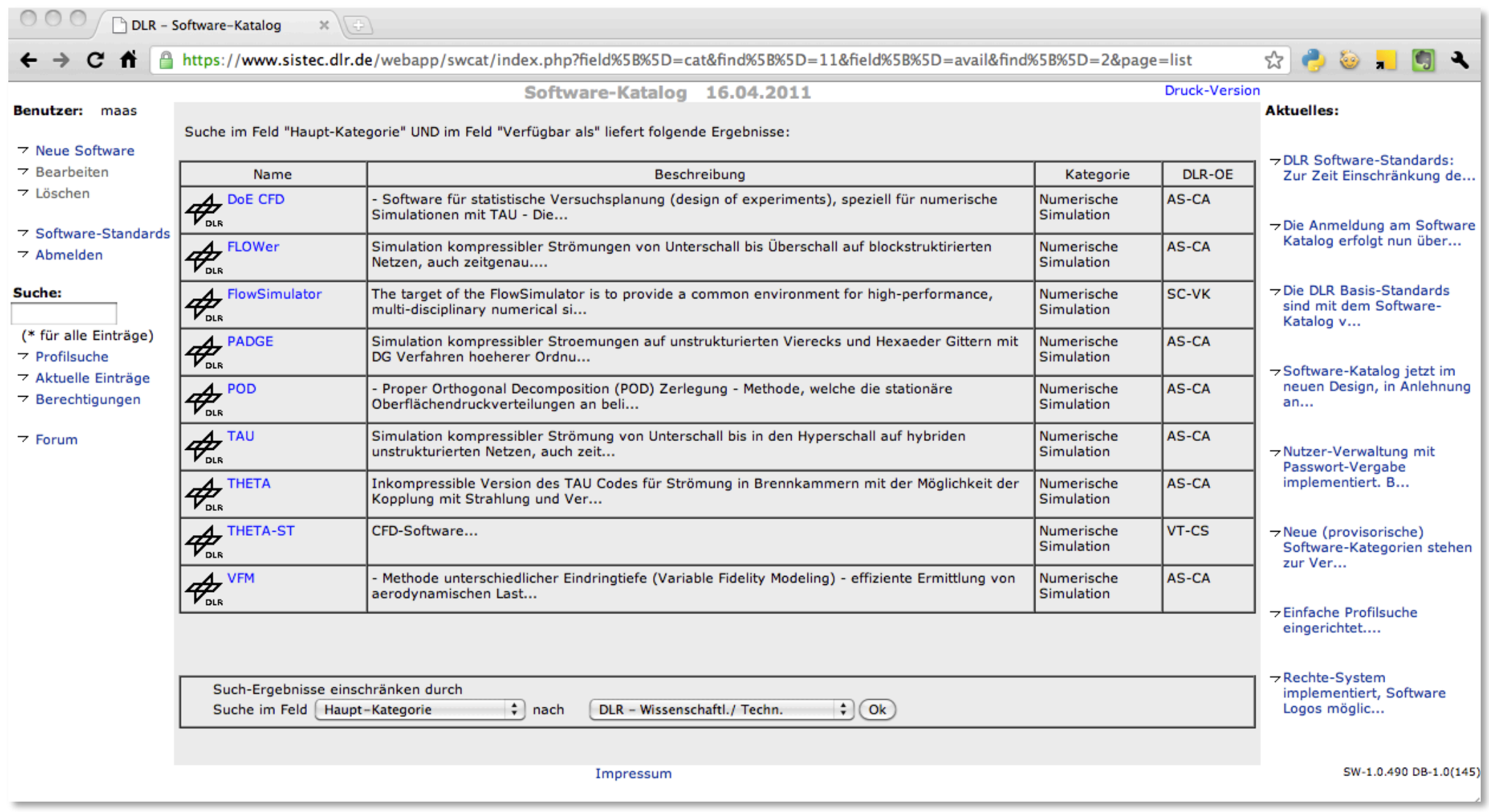




\section{Software Catalogue}

\section{First Version}

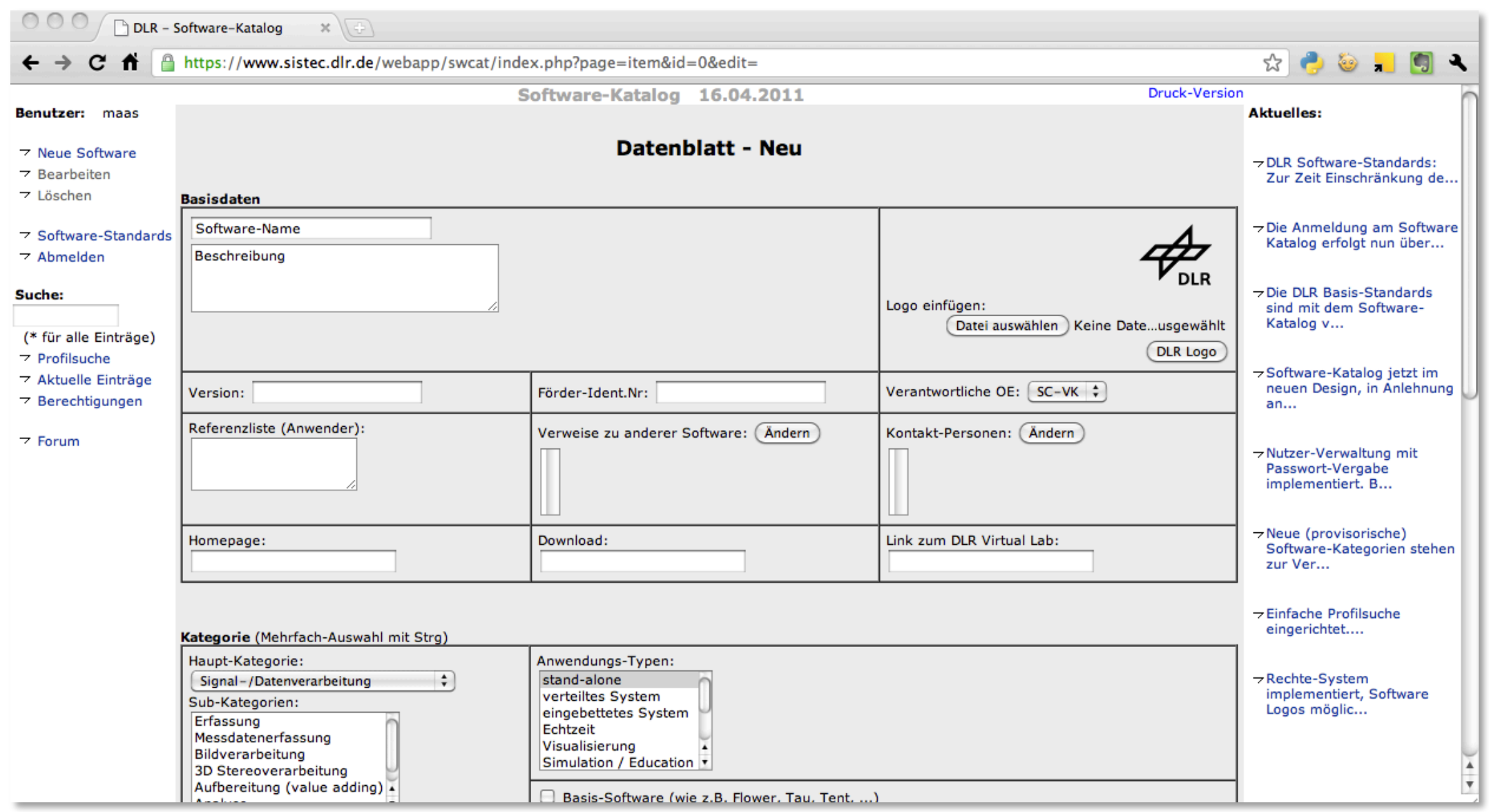




\section{First Version Problems}

\section{Problems of the first version}

- The solution was not ergonomic

- Employee acceptance was low

- Not suitable for the public

- Code (PHP) not very maintainable

\section{Conclusion}

- Development of a new version based on existing Open Source software

$\rightarrow$ Apache Allura

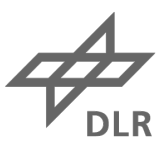




\section{SourceForge.net}

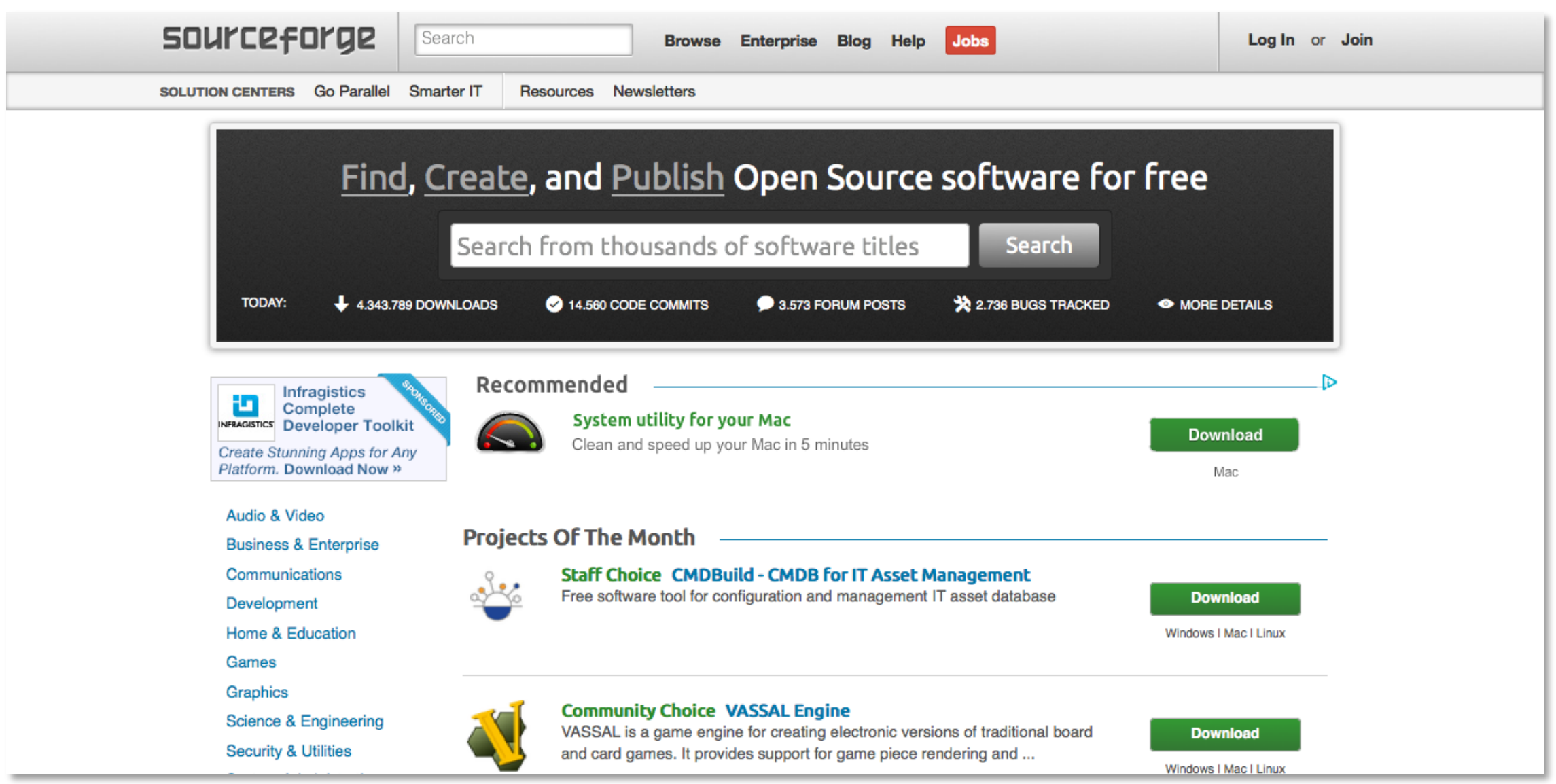




\section{Apache Allura \\ The Software behind SourceForge.net}

„Forge“ implementation

- Source Code Repositories

- Bugs \& Issues

- Discussions

- Mailing Lists

- Wiki

- Blogs

Open Source, Apache project since 2013

- https://allura.apache.org

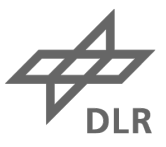




\section{Allura}

https://forge-allura.apache.org

Register Log In

Home / Projects / Apache Allura ${ }^{\mathrm{TM}}$ / Git

Apache Allura ${ }^{\mathrm{TM}}$

Forge software for hosting software projects

The Apache
Software Foundation

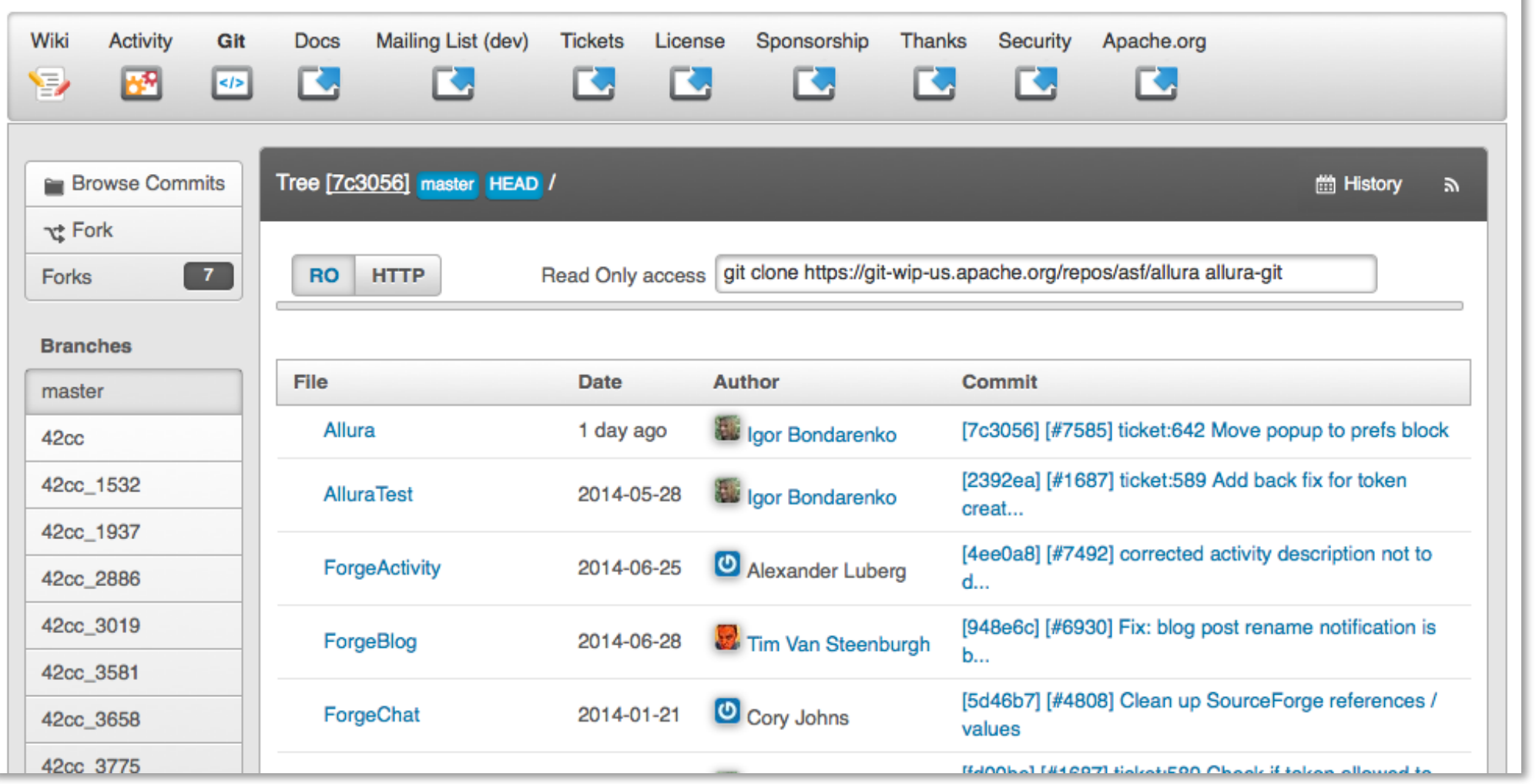




\section{Allura Integrated Tools}
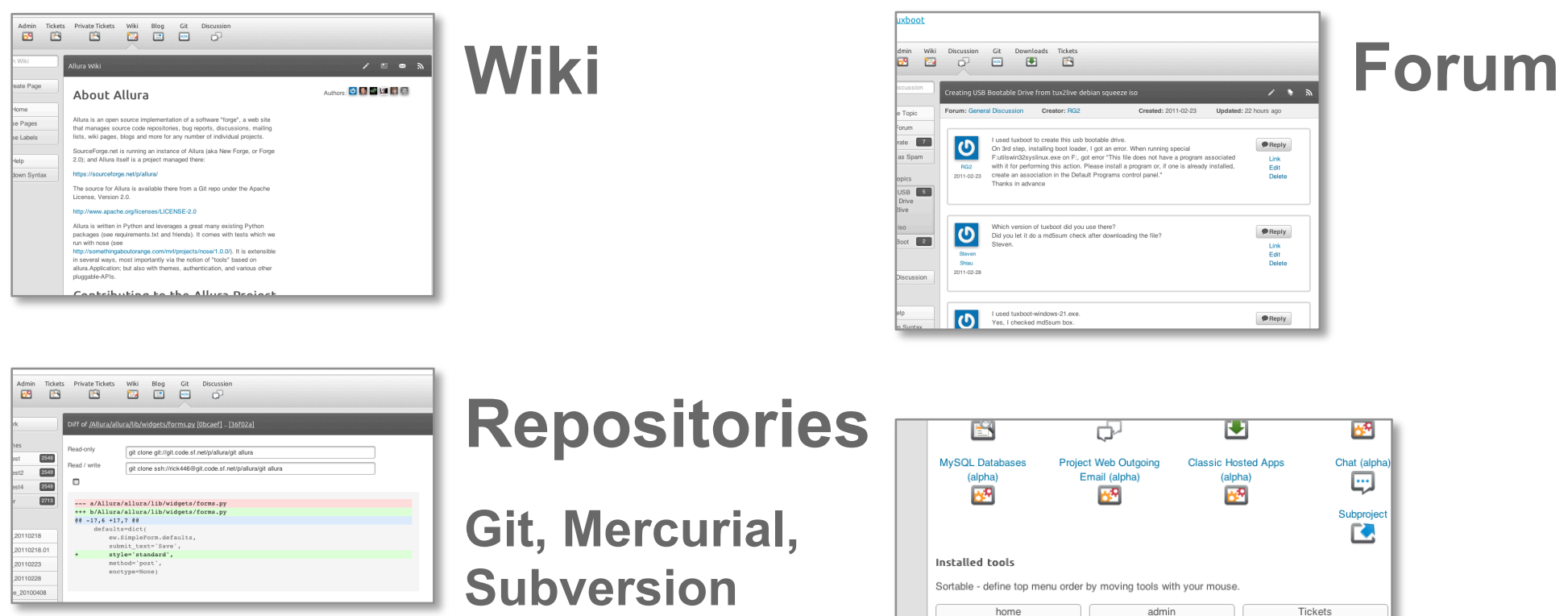

Repositories

Git, Mercurial, Subversion

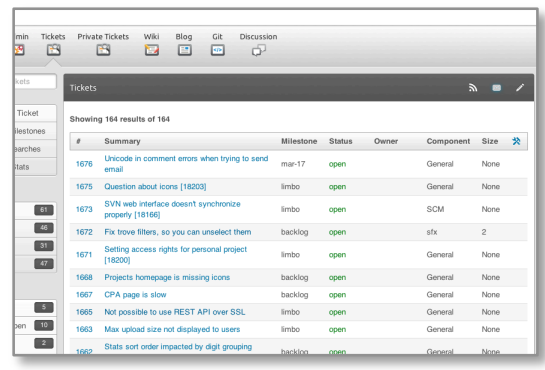

Tracker

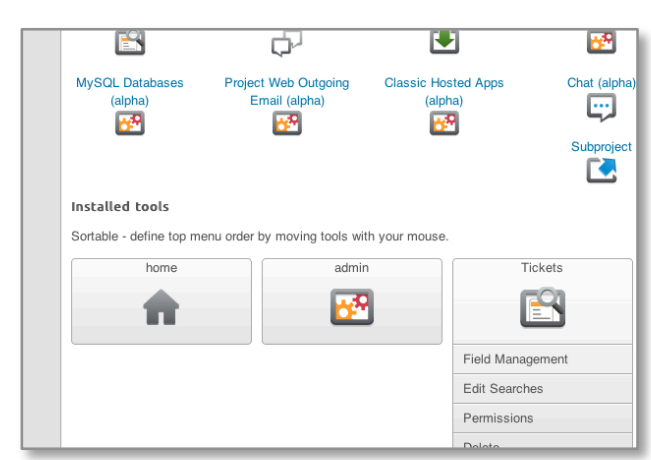

Administration

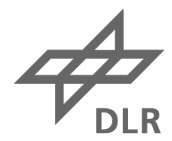




\section{Allura Software Platform Building Blocks}

SMTP

(Incoming)

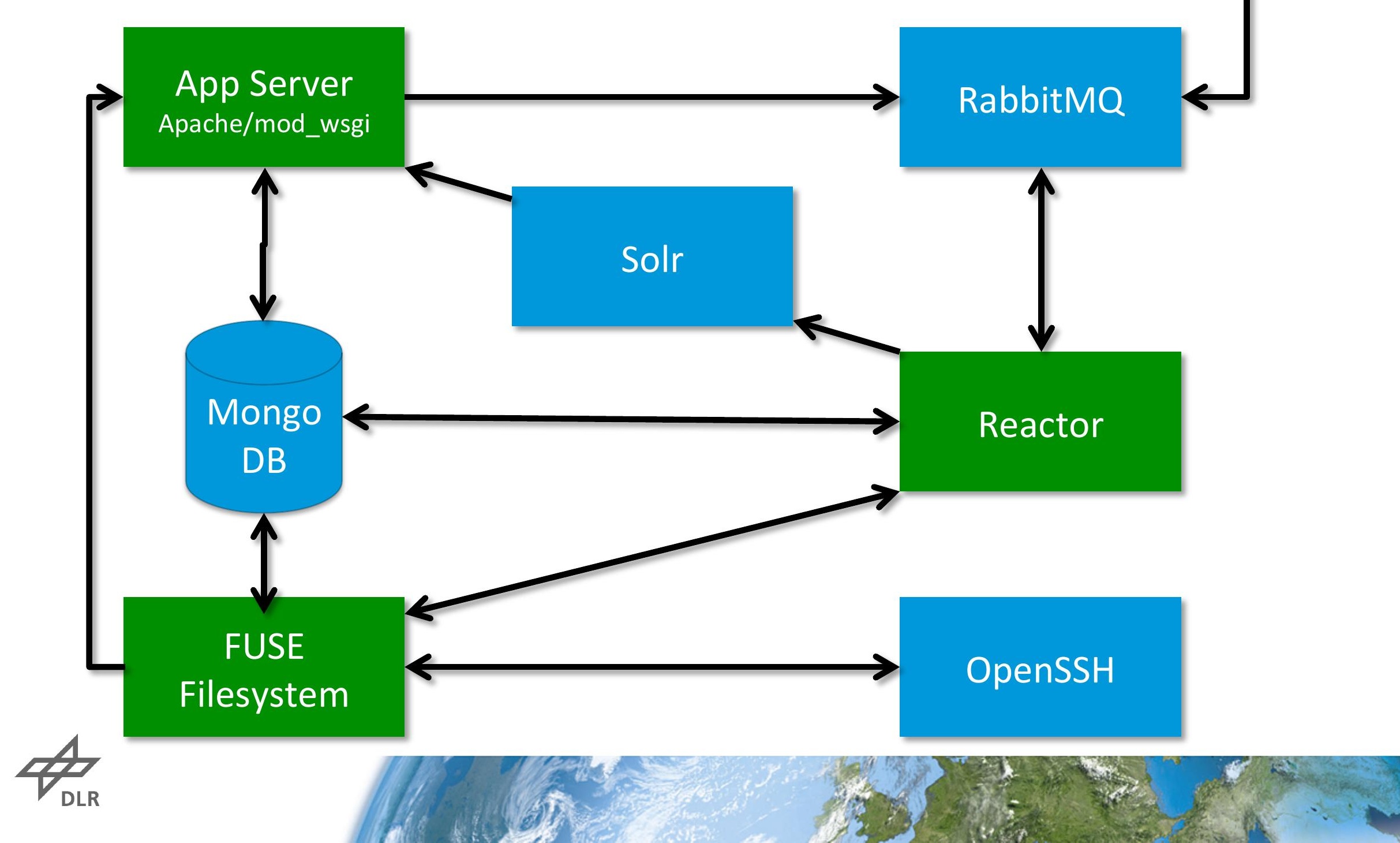




\section{Allura Software}

Components

\section{WSGI Stack}

\begin{tabular}{|c|}
\hline TurboGears \\
\hline Pylons \\
\hline Beaker \\
\hline Paste \\
\hline WebOb \\
\hline
\end{tabular}

Rendering

\begin{tabular}{|c|}
\hline Pygments \\
\hline Markdown \\
\hline Jinja2 \\
\hline FormEncode \\
\hline EasyWidgets \\
\hline
\end{tabular}

Search

$$
\text { PySolr }
$$

\section{Repositories}

GitPython

Mercurial

PySVN 


\section{software.DLR.de}

\section{Follow us}

A RSS Feed

f Facebook

E Twitter
$\Theta$ Administration and Tools

$\Theta$ Communication

\section{$\Theta$ Control}

$\boldsymbol{\Theta}$ Knowledge and Data Management

$\Theta$ Signal and Data Processing

\section{$\boldsymbol{\Theta}$ Software Engineering}

$\Theta$ Simulation and Modeling

$\boldsymbol{\oplus}$ Visualization

\section{Knowledge and Data Management}

\section{BACARDI}

The Backend Catalog for Relational Debris Information (BACARDI) is the DLR's approach to a space debris database. The custom middleware components are implemented in Python using ZeroMQ and Protocol Buffer technology.

.

Simulation and Modeling

\section{Simulation Model Library}

Simulation Model Library (SimMoLib) is a distributed system to manage a library of simulation models. SimMoLib's main goal is to promote the preservation of knowledge that lies in simulation and calculation models and encourage reuse of those models.

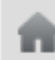

\section{Simulation and Modeling}

\section{Virtual Satellite}

Designing space systems and planning space missions relies on many separated phases and dispciplins. The virtual satellite aims at closing the gaps in the development life-cycle and between disciplins by using model-based systems engineering. 


\section{DLR Software Portal}

http: //software.DLR.de

\section{Basics}

- Development started in 2011

- Available for DLR employees and the public

- For Open Source as well as proprietary software

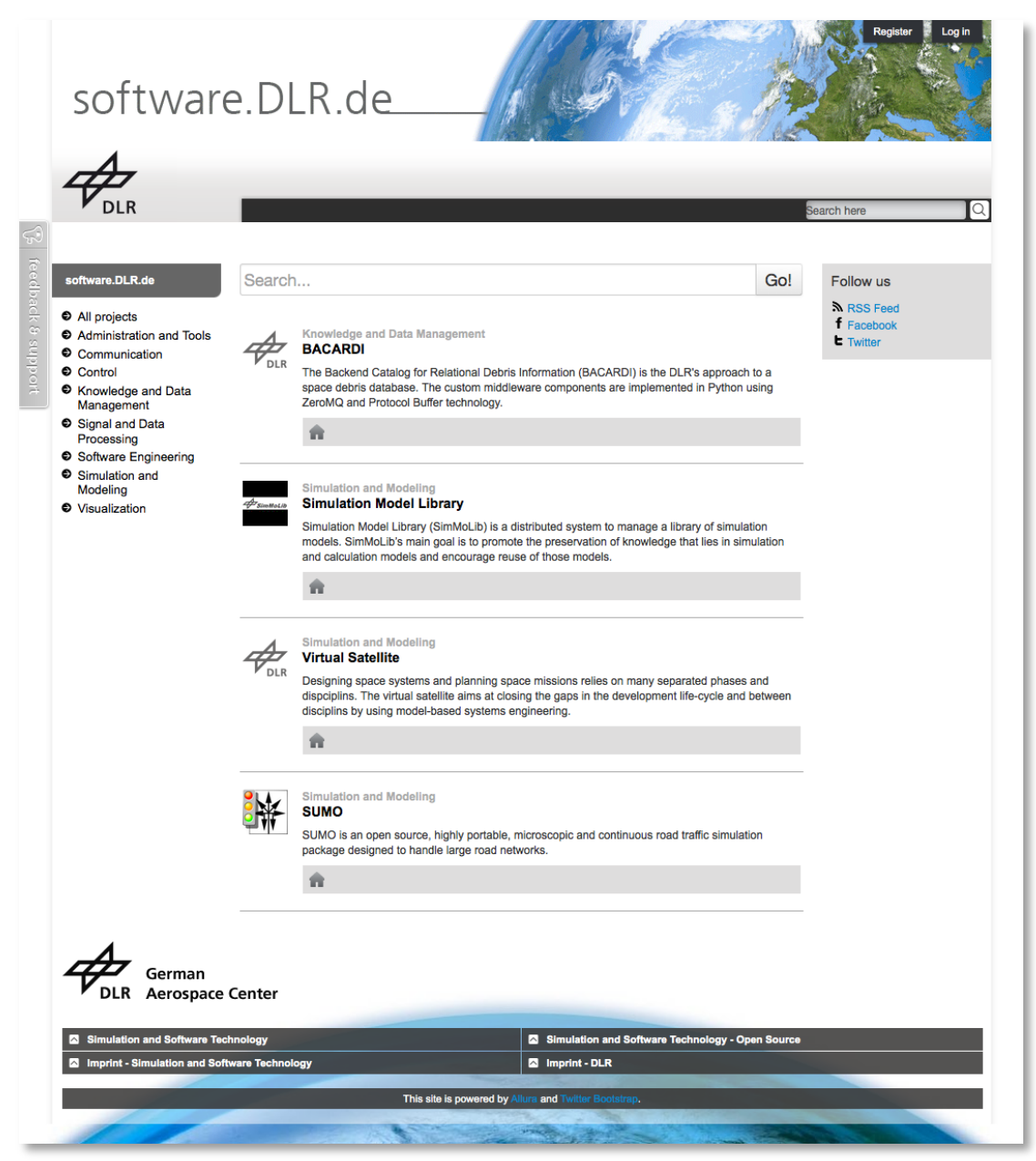




\section{DLR Software Portal Customization}

\section{Customization of Allura}

- Web templates (DLR corporate design)

- Metadata (project overview and basic information)

- Categories

- DLR site

- Development status

- Institute

- License

- Operating system

- Programming language

- DLR research program
$\Theta$ Administration and Tools

$\Theta$ Communication

$\Theta$ Control

$\Theta$ Knowledge and Data Management

$\Theta$ Signal and Data Processing

$\Theta$ Software Engineering

$\oplus$ Simulation and Modeling

$\Theta$ Visualization 


\section{DLR Software Portal Rollout}

Rollout in four major steps

- [2012:] Open to the public for searching and browsing. Access to add entries for two selected institutes of DLR and for selected users. Code hosting is disabled.

- [2013:] Access to every DLR employee for adding entries. Changed layout for project home pages, project editor, and user profile pages.

- [2014:] Extended features for faceted search and browsing.

- [2015:] Code hosting enabled. Access to registered external users (who must have an account at DLR, which is usually given to project partners or students)

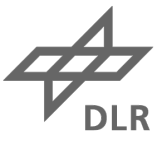




\section{software.DLR.de}

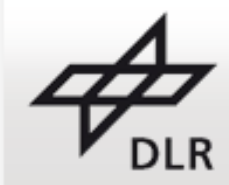

software.DLR.de

» Projects

» TIGL

TIGL

Home

Search here

TiGL

Visualization

TIGLViewer Screenshot
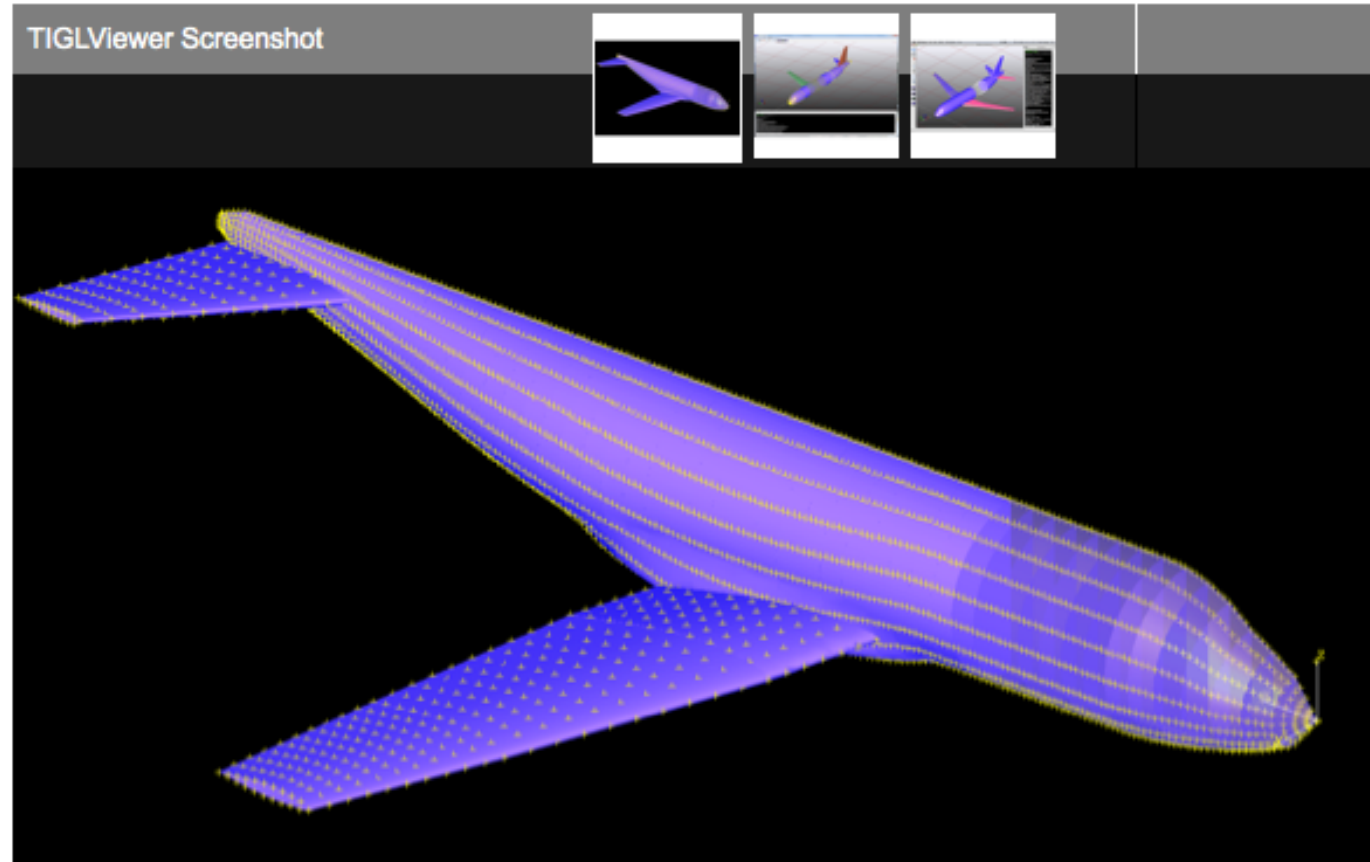

4| 1/3 |

\section{License}

Apache Software License

\section{Programming}

Language

C

$\mathrm{C}++$

Python 2

Fortran

Matlab

Development Status

5 - Production/Stable

Operating System

Windows 7

Linux

OS $\mathrm{X}$

Android

\section{Research Program}

$\mathrm{L}$ - no assignement

Institute

Simulation and Software Technology

The TiGL Geometry Library can be used for easy processing of geometric data stored inside CPACS data sets. TiGL offers query functions for the geometry structure. These functions can be used for example to detect how 
software.DLR.de

" Projects

" TIGL

\section{$\Theta$ Metadata}

$\oplus$ Homepage

$\Theta$ Screenshots

$\oplus$ Categorization

$\Theta$ Permissions

$\Theta$ Usergroups

$\Theta$ Audit Trail

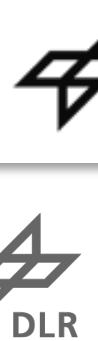

\section{Project Setup}

Please set up and update all information for your project.

Important: Don't forget to set and maintain correct permissions!

\section{Basic Project Information}

Metadata Update basic project metadata, such as project name, links to other websites, a short summary of your project, the software category, and the icon. (Info: You can also remove your project here.)

Homepage Provide a solid description, so colleagues can figure out what the project is all about.

Screenshots Add as much screenshots, pictures, and diagrams as you like.

\section{Categorization}

Categories Categorize your project. Currently, you can categorize according to license, programming language, and DLR research program.

\section{Access}

Permissions Set permissions to groups for reading, updating, administrating or creating project content.

User groups Manage user groups for your project.

History

Audit trail Show all changes on the project information 
software.DLR.de

" Projects

"TIGL

Metadata

$\Theta$ Homepage

$\Theta$ Screenshots

$\Theta$ Categorization

$\oplus$ Permissions

$\Theta$ Usergroups

$\oplus$ Audit Trail

\section{Metadata Project Overview and Basic Information}

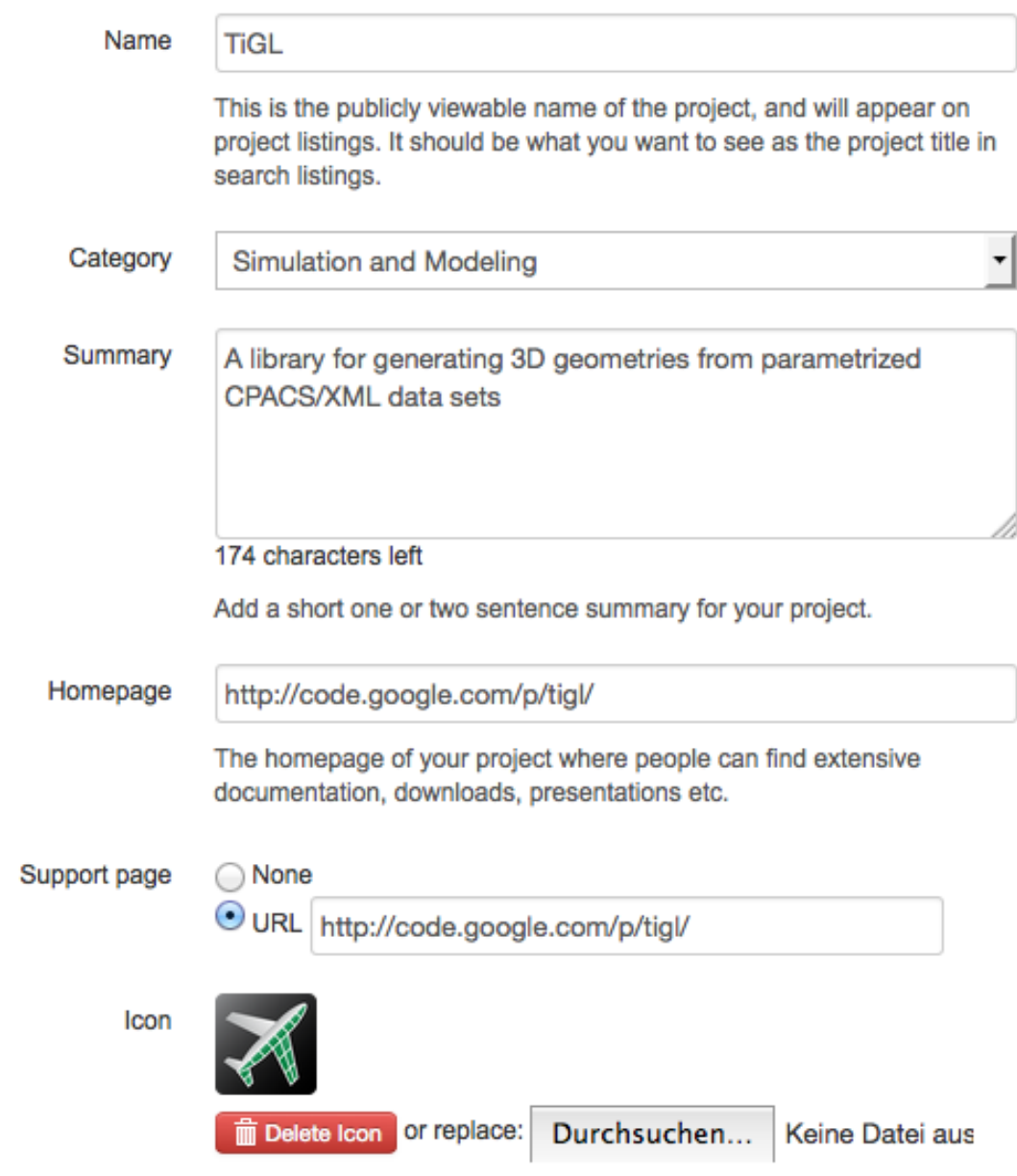

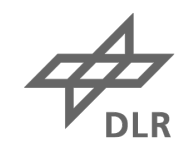


software.DLR.de

" Projects

" TIGL

\section{$\Theta$ Metadata}

$\Theta$ Homepage

$\Theta$ Screenshots

Categorization

$\oplus$ Permissions

$\Theta$ Usergroups

$\oplus$ Audit Trail

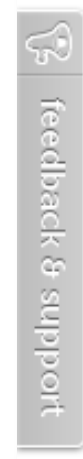

\section{Categorization Categories of the Project}

\section{DLR site}

x DLR site :: Cologne

\begin{tabular}{|l|}
\hline Augsburg \\
Add
\end{tabular}

\section{Development Status}

X Development Status :: 5 - Production/Stable

\begin{tabular}{|l|}
\hline 5 - Production/Stable \\
\hline Add
\end{tabular}

Institute

$x$ Institute :: Simulation and Software Technology

\begin{tabular}{|l|}
\hline Design Organisation \\
\hline Add
\end{tabular}

\section{License}

x License :: OSI-Approved Open Source :: Apache Software License

OSI-Approved Open Source
Academic Free License (AFL)
Add




\section{DLR Software Portal Current State}

- Open for all DLR institutes

- First set of projects added

- Adding projects not mandatory yet

- Feedback by project owners

- Many bugs and feature requests

- New contacts within DLR and with external companies 


\section{DLR Software Portal Current and Future Work}

Technical

- Upgrade to latest version of Allura

- Faceted search

- Activation of code hosting

\section{Organizational}

- Engage DLR employees to add their projects

- Extend access to other organizations (ESA, NASA, ...)

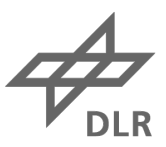




\section{Thank You!}

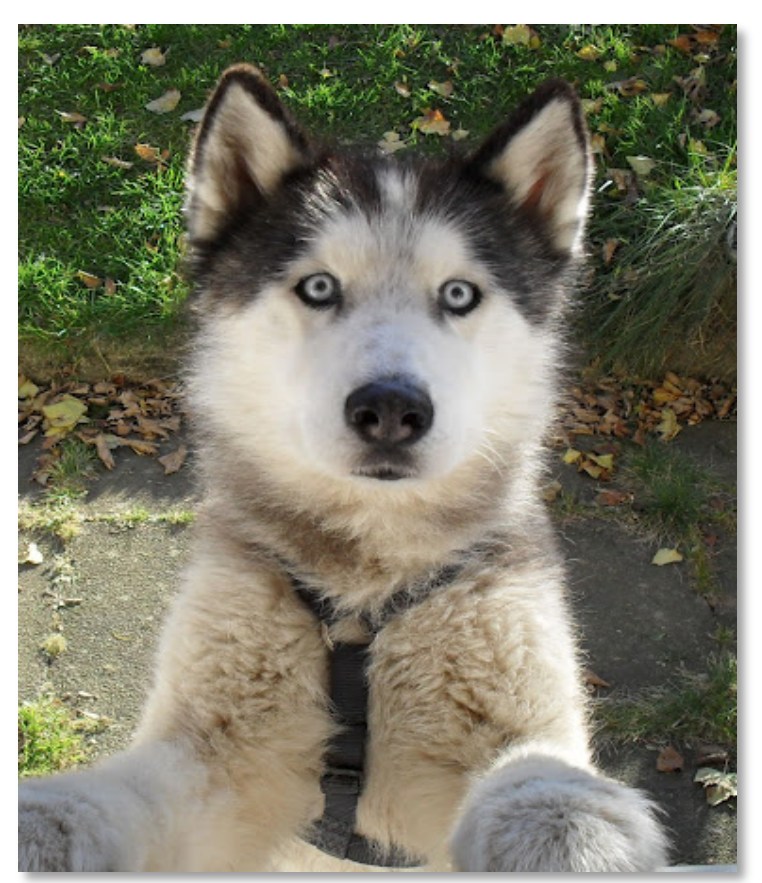

\section{Questions?}

\section{Andreas.Schreiber@dlr.de}

\section{www.dlr.de/sc | QDLR_software | Qonyame}

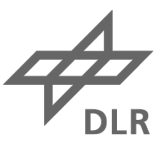

